\title{
Autonomic Computing and Ontologies to Enable Context-aware Learning Design
}

\author{
Patricia Charlton and George D. Magoulas \\ London Knowledge Lab, Birkbeck College, University of London, UK \\ \{patricia,gmagoulas\}@dcs.bbk.ac.uk
}

\begin{abstract}
Semantic web technologies and autonomic computing principles are combined in this paper in an attempt to design and build a learning design environment that possesses context-aware features. Our approach builds on the features of self-management and organisation of autonomic computing but uses self-configuration as a means to extend a knowledge-based inference through the design of meta-level inference. Thus, the context inference is modelled using a meta-interpreter and self-configuration rules. The details of our approach are presented demonstrating the use of self-configurable inferencing to support the creation and use of context-paths across learning design domain concepts. The paths exploit ontology alignment principles to determine contextual relevance between user learning designs and core system knowledge.
\end{abstract}

Keywords - context-aware, instructional design, integration of intelligent technologies, learning design, ontology, self-configuration, semantic web technologies.

\section{INTRODUCTION}

The term "Learning Design" has been in use only in recent years; the earliest work in the field can be traced back to "instructivist" approaches [11]. The development of interest in "Learning Design" as a focus of research began with this recognition that the constructivist pedagogical theories did not easily transfer to the practice of teaching [10]. The emphasis on what learners were doing and how to support their activities was much less constrained by constructivism. Supporting these design principles has required re-thinking how to support learning designers, exploiting the 'constructivist' potential of digital technologies for learning.

In this paper we present a framework for supporting next generation Learning Design (LD) tools and an architecture of. a LD Support Environment (LDSE) that implements this approach. To manage and exploit the semantic of concepts used when creating the learning design we use self-configuration, an autonomic computing technique, which enables us to inference about appropriate context changes, as well managing context alignment via the underlying ontological models.

The paper is organised as follows: In Section II there is a review of learning design tools and limitations. Section III provides the requirements for a learning design environment and evaluates tools with respect to selfconfigurable and context-aware capabilities. Section IV provides a short summary about the background of autonomic computing and context-aware systems and the use of ontologies. In Section V we present our approach to support context-aware learning design. Section VI illustrates the overall architecture and self-configurable inferencing details demonstrating the creation and management of context-paths. Section VII concludes the paper.

\section{LEARNING DESIGN TOOLS}

There is considerable work on developing various languages and formalisms for LD (e.g. [18][19]). The Educational Modelling Language (EML), which appeared in 2000, was the outcome of work that started in 1997 by the IMS Global Consortium (IMS) and the OUNL. Initial work by the IMS targeted support processes for learning rather than the learning process itself but by early 2001 it was realised that a specification was needed to describe the learning processes. The EML approach to pedagogy is to provide a high-level abstraction of learning methods, including actors (e.g. tutors and students) and roles (e.g. activities) undertaken in an environment. The term "environment" has been used in EML to describe learning content, tools, communication, and other elements usable by learners and others in an activity. Activities are structured using a "learning flow" that includes decisionpoints (so that, for example, performance in one activity determines the next), sequences and choices. The EML focused on the entire learning process and was considered as complementary to the specifications developed by IMS $^{1}$.

There have been several attempts in this area internationally, most notably the PALO language (http://sensei.ieec.uned.es/palo/) and the E2ML [15]. The PALO approach allows creation of a course-specific repository of semantically linked material rather than a set of local or distributed knowledge objects, which leads to

\footnotetext{
${ }^{1}$ The IMS LD 1.0 adopted the XML format, which is not visible to the designer but works behind the scenes like the converters of document formats used in software applications.
} 
the construction of a knowledge base that is organised along a set of themes/learning scenarios. In E2ML, goals, requirements and design of the teaching and learning activities are described in a visual language. The E2ML model is compliant with the IMS LD specification.

IMS LD have motivated developments in authoring using tools that exploit IMS LD concepts, such as the Unit of Learning (UoL), or are IMS LD compliant ([16]). Some examples are editors like CopperAuthor ${ }^{2}, \mathrm{Cosmos}^{3}$ or Reload LD Editor ${ }^{4}$, which can be run with several tools and engines, like CopperCore ${ }^{5}$ or Sled ${ }^{6}$. However, current tools are not very friendly to non technical users as they assume that the teacher knows the technical editors and the specifications in depth. Paquette's work uses OWL (the Web ontology language), as a key component in developing formal representations. This work can inform the development of next generation of environments for learning design by matching it with design-based representations that mesh with and extend effective teaching practice.

\section{MOTIVATION FOR OUR APPROACH}

The main point behind the approach presented in this paper is that although LD information can be quantified for engineering purposes, as done in the works mentioned above, only LD knowledge is of real social and economic importance and can help to increase the rate of adoption and change current teacher practices. This kind of knowledge must be assimilated by humans before they can use it and is not enough to copy information or reproduce learning design products mechanically using XML, Petri Nets or LAMS sequences. LDSE works more like a system to manage tacit knowledge (i.e. knowledge acquired from practical experiences). This type of knowledge cannot be easily formalised (e.g. using Petri Nets) and not a single actor knows the whole picture. Thus it is hard to learn and pass on and this knowledge has not yet been given sufficient recognition in the approaches to $\mathrm{LD}$ so far. However, this form of knowledge is an important component of the teaching profession, and social cooperation and a common understanding are crucial to task performance. Moreover it is an essential part of the educational environment and affects its economic performance.

Current Learning Design (LD) tools described in Section II can be roughly organised into four groups (a) standards-based, (b) generic form-based, (c) authoring tools and (d) ontology-based; see [20] for an overview of tools and their properties. We can briefly say here that standards-based approaches, such as EMLs and IMS-LD [2], provide greater interoperability between tools and designs. It enables building tools with specific

\footnotetext{
${ }^{2} \mathrm{http}: / /$ sourceforge.net/projects/copperauthor/

${ }^{3} \mathrm{http}: / /$ www.collide.info/Members/admin/publications/ICCE05.260.pdf

${ }^{4} \mathrm{http}: / /$ www.reload.ac.uk/

${ }^{5} \mathrm{http}: / /$ coppercore.sourceforge.net/

${ }^{6} \mathrm{http}: / /$ sled.open.ac.uk/
}

functionality, e.g. LAMS $^{7}$, Moodle ${ }^{8}$. They facilitate creating activity sequences, supporting from technical point of view modeling of various design methods, theories and approaches and generating designs through Learning Design engines. Generic tools, such as Phoebe ${ }^{9}$ and CompendiumLD ${ }^{10}$, are used for designing, managing and delivering learning activities and content, e.g. learning design documents and, in certain cases, enable collaboration, online learning and social networking (e.g. Cloudworks ${ }^{11}$ ). Authoring tools usually support a particular instructional design theory and employ inference engines that enable sequencing and presentation of instructional material depending on learner characteristics; an overview is given in [4]. Ontologybased tools represent domain concepts and relationships [5] as well as educational theories and relationships [3] among them. They facilitate communication and sharing of LD knowledge through common vocabularies and the development of a rational for learning designs use, modeling, and most of the time promote a particular pedagogy approach.

However, these tools do not support the context-aware needs for complex applications and they are limited in their adaptation to user interaction. While context-aware [8] is often classed as event-driven, here we mean datadriven or more precisely domain knowledge-driven in the sense that it depends on learning designer's practical knowledge and perception of the domain of LD and process of designing for learning. In terms of adaptation we refer to the application having some degree of configurability by using the knowledge-aware context. While this builds on the type of features of selfmanagement and organisation that is expressed in autonomic computing [1], here we use self-configuration as a means to extend the knowledge-based inference through the design of meta-level inference. This provides a knowledge-aware application with flexibility in finding, using and presenting to the user appropriate Learning Design content.

Our approach has been designed to support multiple theories about LD, which assists in creating application context, by employing an ontological model. This has been developed based on interviews with $L D$ practitioners and LD case studies (see [7] for a full description of the methods used to construct our approach) and is theoretically underpinned by the Conversational Framework (CF) [12]. CF provides conceptual depth and perspective round a number of pedagogical theories with a clear mapping of a unit of learning into the broader ideas of a constructivist perspective. This has led to an ontological design of the system, where a unit of learning may differ both in concept and content to the work of others, such as Mizoguichi [13] who has also attempted to create a theory-aware environment by focusing on a

\footnotetext{
${ }^{7} \mathrm{http}: / /$ www.lamsinternational.com/

${ }^{8} \mathrm{http}: / /$ moodle.org/

${ }^{9} \mathrm{http}: / /$ www.phoebe.ox.ac.uk/

${ }^{10} \mathrm{http}: / /$ compendiumld.open.ac.uk/

${ }^{11} \mathrm{http}$ ://cloudworks.ac.uk/
} 
particular instructional design theory. Moreover, our design approach to being both theory-aware and contextaware is the inclusion of self-management functions as part of a knowledge inferencing mechanism. Contextawareness is generated based on information from user choices during the process of LD and allows the system to provide appropriate concepts, which are relevant to the current user context. Our approach leveragea the underlying ontological model and relationships, allowing the provision of context-aware support during the LD process.

\section{COMBINING AUtONOMIC COMPUTING AND ONTOLOGIES FOR CONTEXT-AWARENESS}

The characteristics often attributed to an autonomic system are a self-managing, autonomous and ubiquitous computing environment that completely hides complexity, thus providing the user with an interface that exactly meets his/her needs [1]. Autonomic computing for LD faces two design challenges: (a) what context to use for self-management and (b) how to collect that particular context in both form and content. Several researchers [6] [8] have tried to categorise context-aware applications according to subjective criteria. A taxonomy on contextaware features is proposed in [6]. There are three general categories of context-aware features that context-aware applications may support [8]: (a) services to a user, (b) automatic execution of a service, and (c) tagging of context to information for later retrieval.

In the LD environment, a system can be considered to be context-aware if it can extract, interpret and use information of the current context of use and adapt its functionality to the needs of the learning designer. Context-awareness when designing for learning allows the LD system to maintain and enhance its representation of the LD domain through automatic or semi-automatic (i.e. user driven) tagging of the learning designs produced and of their elements, trigging configuration rules that self-adapt and self-organise the underlying concepts of a learning design as changes take place by the user. The context, which is represented as a set of concepts that are used to build a context-path, can provide the relevant knowledge at run-time for the user. There are two ontological models held by the LDSE system that serve this purpose. The first is the LDSE core ontology and the second is created at run-time when the user is creating or re-using learning designs, which may include modifications to the concepts that are part of the core ontology. As these modifications take place a meta-level inference is used to investigate the changes, e.g. monitoring whether the concepts modified to match users workflow, when creating and re-using learning designs, are close enough to existing core concepts; supporting the user at various decision points of the LD process.

The self-configurable inference is about supporting "individual" contextual information, from history of use or declared alternative terms, or modified properties of learning designs. For example, the individual contextual information can come from re-using existing learning designs, when creating personal terms from the common set of terms and refining the properties of common concepts that exist in the core ontology.

\section{SELF-CONFIGURATION APPROACH AND USE OF ONTOLOGIES}

Self-configurable rules are used by the LDSE to assist in pushing LD concepts and learning designs that match user's current context of use. The context is gradually constructed by concepts included in the user's learning design. These either refers directly to concepts in the core ontology or are, new concepts and content that are added by the user. Thus they cannot be recognised as part of the core ontology and are "indirectly" referenced. The selfconfigurable context is built as a path. It is created as a conceptual network. Concepts and properties are matched in terms of similarity measure. The similarity measure provides constraints in determining, which cluster of concepts best reflect the creation of a learning design.

\section{A. Constructing a Context Path}

When the LDSE receives a new input through from user interaction, it needs to reason about this user input and set up a context path that reflects its understanding of the learning design being created. This context is passed to the inference configuration and the necessary computations needed to deal with the input are created. Once a concept has been added to the context path then the management of the path requires handling further modification to properties and deletion of concepts. These changes require a call to configure inference again with type similarity-context or same or indirect-context. However, it is possible that a configuration can result in a context-path being unbounded or highly fragmented. Essentially, the set of concepts changes made by the user are such that the mapping to the core ontology is limited, and thus limited knowledge can be inferred from the context-path related to the current learning design.

\section{B. Managing the Context Path through Ontology Alignment}

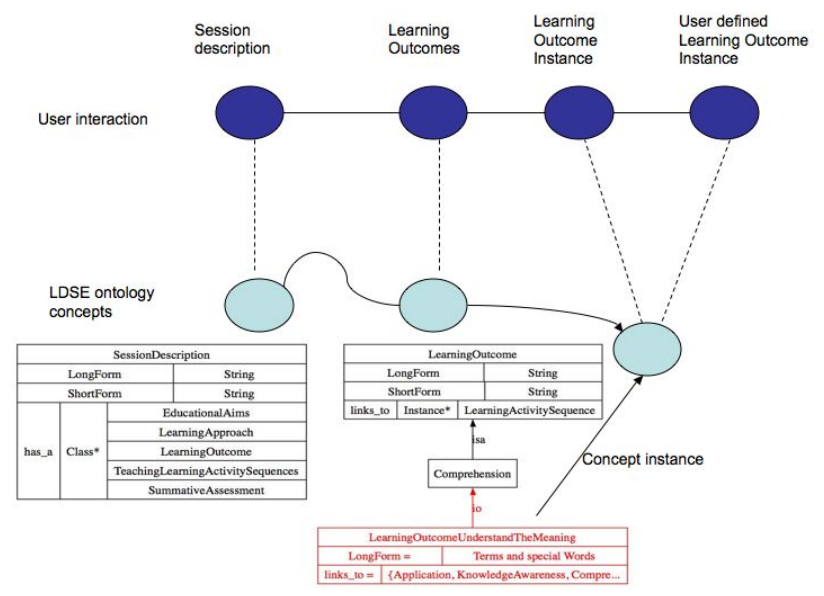

Figure 1. An example of ontologies and context creation that occurs when a user creates a simple session. 
Within the LDSE the core ontologies are known, possible extensions are enabled and although general extensions are possible through the self-configurable approach the ontology extension is scoped. The creation and management of the context-path is considered a simplified form of ontology alignment, which is described as the task of finding relationships holding between the entities of two different ontologies; thus establishing a set of mappings expressing the correspondence between two entities of different ontologies through their relation

In the LDSE system there are two context-paths created. The first path contains the original core LDSE ontologies and the second path the LDSE ontologies used, modified concepts and the user-created ontologies. Both context-paths form part of the formal semantic tagging of the learning designs. This means that when the editing, or sharing or re-use, of a design happens ,the underlying concepts and the context in which they were used can be elicited to support the creation of further learning designs. Fig 1 provides an example of using the LDSE to create a learning design using the ontology. This also illustrates the creation of a user context path and an LDSE context path that are aligned through the core ontology. The ontology alignment in LDSE is specialised to a set of correspondences between two ontologies, which are expressed as mappings between two context-paths as well as within a context path. The mapping within a contextpath is a formal expression that states the semantic relation between two entities belonging to different ontologies. However, mapping between two context-paths provides one ontology as an extension or modification of another, and thus the similarity-measure becomes an evaluation of the extension or modification to use both the initial ontology and the user ontology edits to see if other core ontology may better serve to underpin the current learning design. The mappings are based on terminology and conceptual similarities. As the users are creating their learning design, some of the original concepts in the core ontology are modified, or new unknown concepts are added. To this end, the concepts that are the same, modified or new are matched to the core ontology by using terminology and conceptual similarity. The result of this matching is used to generate the context-path of the learning design. Knowing the concepts that are similar is used to configure the next set of appropriate concepts for the user, providing better support as more contextual information becomes available..

Terminological similarity is the part of a mapping that expresses terminological relations between the lexical expressions used to name the entities to be mapped. Simple examples are: the name of two entities is the same, the name of an entity is an abbreviation of the name of the other or has been created by the user through the pedagogy thesaurus and contains same properties but different names of the concepts.

Conceptual similarity is the part of a mapping that expresses the relation between entities in different ontologies. Simple examples are: concept $\mathrm{c}_{1}$ in ontology
$\mathrm{O}_{1}$ is equivalent to concept $\mathrm{c}_{2}$ in ontology $\mathrm{O}_{2}$, concept $\mathrm{c}_{1}$ in ontology $\mathrm{O}_{1}$ is similar to concept $\mathrm{c}_{2}$ in ontology $\mathrm{O}_{2}$, instance $i_{1}$ in ontology $O_{1}$ is the same as instance $i_{2}$ in ontology $\mathrm{O}_{2} . \mathrm{O}_{2}$ is built as the user creates their learning designs and may never differ from the original core ontology $\left(\mathrm{O}_{1}\right)$ except in values and terminology. However, extensions and modifications and deletion of properties are possible by the user and the inclusion of non-core concepts means that handling the context of similarity and indirect mappings is required. This means:

- given two ontologies $O_{1}$ and $O_{2}$ with different coverage, tells us how the two ontologies can be used together to achieve a (less partial) description of the LD domain in LDSE. This permits clustering relevant concepts when the user is creating, editing or sharing a learning design.

- given two ontologies $O_{1}$ and $O_{2}$ with different granularity, tells us how facts in $O_{1}$ can be systematically translated into facts of $\mathrm{O}_{2}$ (for example, how a fact $f_{l}$ belonging to $O_{l}$ can be rewritten as a logically equivalent fact $f_{2}$ in $O_{2}$ ). The user may chose a particular instance but wish to use a different set of terms. If the properties remain the same then the LDSE can draw from the original concepts and relationships to provide relevant information.

- given two ontologies $O_{1}$ and $O_{2}$ with different perspective, tells us how a fact $f_{l}$ in $O_{l}$ would be seen from the perspective of $\mathrm{O}_{2}$.

Over time the context-path itself provides the contextual cues in determining a user ontology alignment relative to the core ontology, e.g. through indirect context, frequency of use of certain terminology and concepts etc. The expression of alignment, simplified by this application, enables LD concepts adapted by the user to be linked to the LDSE core ontology. The use of "with different perspectives" alignment enables the provision of a pedagogy framework, where the use of user terminology within the creation of a learning design can be "aligned" with the underlying concepts in the LDSE, supported by LD relationships.

Fig 1 provides an example of ontologies and context creation that occurs when a user creates a simple session with a learning outcome using the LDSE ontology core concepts. In the knowledge-base there is a basic concept about a session. Each session will have a description and is ideally expected to have at least one other concept (indicated by the has-a relationship). The other concept can be, for example, educational aims, learning outcomes, learning activity sequence or summative assessment. In this example the user has chosen the learning outcomes concept and a set of learning outcomes (in LDSE these are based on Blooms Taxonomy) are presented. Each learning outcome concept has a set of instances (an instance may also belong to a number of learning outcome concepts,) e.g. "Comprehension" is a kind of learning outcome. Furthermore, "a Learning Outcome" is an 
abstract concept, which has a relationship links_to "Learning Activity". For the expert the concept "Learning Activity" has details of achieving a particular learning outcome, which requires a relationship achieves from "Learning Activity" "Learning Outcomes". Learning Outcomes also have relationships defined indirectly with session types and more directly with the learning approaches. Within certain concepts, properties are themselves concepts and so at any one interaction point with the user the LDSE inference engine can draw from the Knowledge-base (KB) a set of appropriate concepts to be used to help in the creation of a learning design. This is the common use of $\mathrm{KBs}$ and inferencing to create inference-based contextual information. However, combining both the $\mathrm{KB}$ and self-configuration we can consider the broader scope of the LD context.

\section{Self-configuration and Inference}

Designs that include meta-interpreters and reflective techniques have been applied to enable the modeling of code as data that can be later included in the system execution. It is a highly compelling technique in distributed systems and is used in various ways to enable users to download and automatically install software, where configurations of the software to the hardware are possible. We use this technique in LDSE to enable selfconfiguration as part of the inference steps about the learning design application. The contextual knowledge gathered as a learning design is created can be used at each inference stage to push appropriate concepts. Within the LD context the concept of "self" can be coarsely divided into:

- The ability to handle high-level tasks and to automate the completion of these tasks. The possible types of knowledge are pre-defined (but not necessarily all instances of the knowledge) and the system has methods, rules and protocols to deal with the automation of tasks;

- Self-management inferencing to determine the next action, when not all of the decision points have been predefined and encoded.

The first issue can be handled by leveraging an ontological model that captures the relationships of learning design. The designs can be tagged with formal concepts in the KB. The second representation of "self", where an inference has the possibility to incorporate selfmanagement, is useful during the evaluation of a LD or when re-using LDs.

\section{LDSE ARChITECTURE: CONTEXT-AWARENESS AND SELF-CONFIGURATION FEATURES}

Fig. 2 provides an illustration of the main components of the LDSE. The conceptual model is represented by the LD ontology and includes relationships that support the use of pedagogy theory in practice. The pedagogy thesaurus permits the user to define their own terms that are linked to the original concepts found in the LD ontology. The usage of the new terms and change to properties triggers a contextual cue analysis to see if there are closer matching concepts than the original concept used. The user model and preferences relate to frequently used LD knowledge and re-use, such as concepts and terms from the pedagogy thesaurus or the learning design ontology. The contextual information generated through the interaction with the system is stored keeping the context path details for later use, e.g. when editing a design. The learning designs that have been created are stored including the relationships and changes made from the original learning design ontology, concepts developed in the pedagogy thesaurus and other contextual information from the user model and preferences. The learning designs are automatically tagged by the system with the appropriated instances and modifications from the learning design ontology. The community knowledge contains the content to be shared and how this is shared and the re-use of other learning designs that contain the learning design tags.

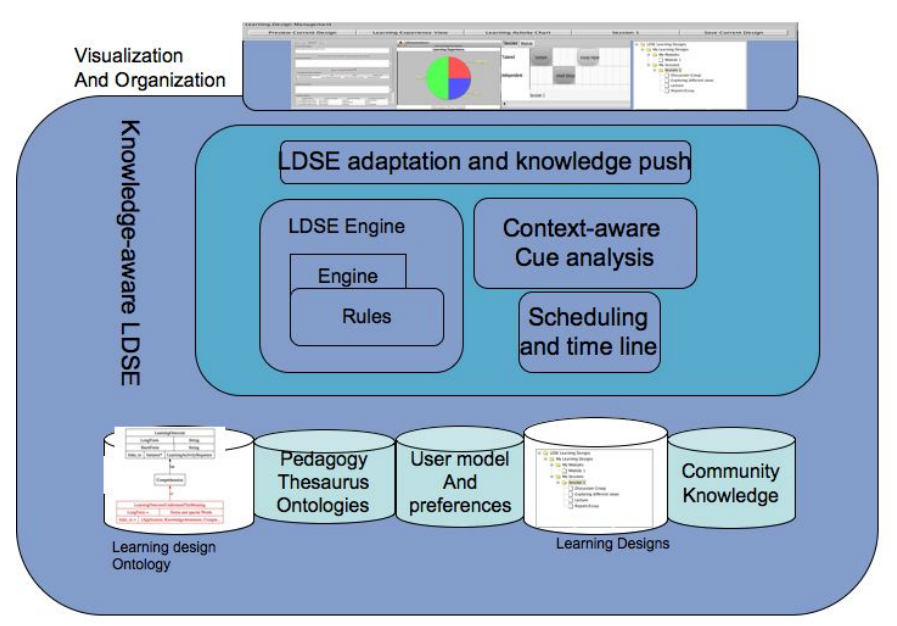

Figure 2. Overview of context-aware LDSE architecture

Key to the design in order to enable both direct mapping and indirect mapping of contextual knowledge is the context path creation and management. As mentioned earlier there are two views of the context path maintained: the expected set of concepts from the core ontology and the modified set of concepts. There is also during the process of creating a learning design additional knowledge generated and, thus, associated with the context, e.g. preferences and particular content. The management of the context path is through the use of a self-configuration system [20]. This enables both flexibility and context inspection at run-time; thus enabling the design adaptation and personalisation.

We use the principles of self-configuration to provide the framework in which the context path can be created. This allows flexibility of design for adaptation through changing concepts, modifying concepts and creating concepts. The inferencing rules through self-configuration and the context path provide the necessary knowledge to manage two context paths and use this to generate semantic tags that take into account the user choices, modifications and original concepts from the domain. 
This permits both the creation and use of a pedagogy thesaurus, which is user-driven, and user preferences. The preference model is built from frequency of use of the concepts and properties based on particular original concepts used and any modification of these concepts. Some of the concepts used by the user are "linked" by interaction only. That is the user generates a particular sequence to create their design. Other concepts are directly drawn from the domain concepts and are part of the initial LDSE context path, which is part of the core ontology. Other concepts are drawn from the pedagogy thesaurus, which links back to the original concepts. The preference concept may not link to the domain knowledge directly but is situated in a context of use and, thus, can be inferred through using the particular knowledge that is located near to the content. The sameness of a concept is determined by the context path and is based on the ontology alignment definitions given in Section V. The knowledge is always driven by the original core concepts but through the use of self-configuration the pedagogy thesaurus or preferences can be taken into account, and the creation of new or modified learning designs are adapted appropriately both with recommendations and automatic tagging by the system.

The current implementation of the LDSE, user interface and integration of the different components is in Java. Protégé has been used to develop the LD domain ontology and JESS has been used to implement the inference engine and core functions of the selfconfigurable framework and contextual cues.

\section{CONCLUSION}

Next generation Learning Design tools and applications have similar design requirements as intelligent applications that create, share and re-use services through the use of data specifications or formal models. Through leveraging the formal semantics of ontological models and inferencing techniques our approach illustrates some of the key cues for enabling context-aware computations that provide intelligent functionality. Our framework adapts autonomic computing principles to create context paths, linking together domain concepts to keep track of the context in which learning designs are created. Such context paths can then be used for a multitude of purposes such as LD reuse and adaptation. This approach could lead to the design and implementation of a next generation learning design tool that is context-aware supporting both knowledge push and knowledge pull to enable appropriate use of theory and practice when generating learning designs for use in higher education. A preliminary evaluation of the context-aware features has been conducted with a small group of users producing promising results for their effectiveness in supporting learning designers in practice. Our work in this area is ongoing and will report further results in the near future.

\section{REFERENCES}

[1] R Steritt, M Parasgar, H Tianfield and R Unland, "A Concise Introduction to Autonomic Computing", Advanced Engineering Informatics, vol. 19, pp. 181-187, 2005.

[2] R. Koper, and Tattersall (Eds), Learning Design: A Handbook on Modeling and Delivering Networked Education and Training, Springer, 2005.

[3] B. S. Barn, "Conceptual Modelling of Educational Theories: An ontological approach", in Proceeedings of the IADIS International Conference on Cognition and Exploratory Learning in Digital Age, pp. $45-51,2006$.

[4] K. A. Papanikolaou and M. Grigoriadou, "Building an Instructional Framework to Support Learner Control in Adaptive Education Systems", in G. D. Magoulas and S.Y. Chen, Eds. Advances in Web-based Education Personalized Learning Environments, IGI Publishing, 2006, pp.127-146.

[5] S.R. Heiyanthuduwage and D.D. Karunaratna, "An Iterative and Incremental Approach for e-learning Ontology Engineering", International Journal of Emerging Technologies in Learning, vol. 4(1), pp. 40-46, 2009.

[6] G. Chen and D. Kotz, "A Survey of Context-Aware Mobile Computing Research", Technical Report TR2000-381, Department of Computer Science, Dartmouth College, Dartmouth Computer Science, November 2000.

[7] P. Charlton, G. Magoulas, and D. Laurillard, "Designing for Learning with Theory and Practice in Mind". In Proceedings of the Workshop "Enabling creative learning design: how HCI, User Modelling and Human Factors help", part of AIED 2009 Conference, pp. 52-61, 2009.

[8] A.K. Dey and G.D. Abowd, "Towards a Better Understanding of context and context-awareness", Georgia Institute of Technology, College of Computing, Technical Report GIT-GVU-99-22, 1999.

[9] R. Mizoguchi, and J. Bourdeau, "Using Ontological Engineering to Overcome AI-ED Problems", International Journal of Artificial Intelligence in Education, vol. 11(2), pp. 107-121, 2000.

[10] D.H. Jonassen, "Thinking technology: Toward a constructivist design model", Educational Technology, vol. 34(2), pp. 34-37, 1994.

[11] C.M. Reigeluth, "What is Instructional-Design Theory and How Is It Changing?", in C.M. Reigeluth, Ed, Instructional-Design Theories and Models: A New Paradigm of Instructional Theory. Mahwah, NJ, Lawrence Erlbaum vol. II. pp. 5-29, 1999.

[12] D. Laurillard, Rethinking University Teaching. A conversational framework for the effective use of learning technologies. Routledge, London, 2002.

[13] R. Mizoguchi,, Y. Hayashi, and J. Bourdeau, "Inside TheoryAware Authoring System", in Proceedings of the $5^{\text {th }}$ International Workshop on Ontologies and Semantic Web for E-Learning (SWEL'07), pp. 1-18. Marina del Rey, CA, USA, July. 9, 2007.

[14] A. Waterson and A. Preece, "Verifying ontological commitment in knowledge-based systems", Knowledge-Based Systems, vol. 12, 12, pp. 45-54, 1999.

[15] L. Botturi, "A Visual Language for Instructional Design: Evaluating the Perceived Potential of E2ML". Proc. Of EDMEDIA 2004, Lugano, Switzerland.

[16] D. Hernández-Leo, "Reusing IMS-LD Formalized Best Practices in Collaborative Learning Structuring", Advanced Technology for Learning. 2(4) 223-232, 2005.

[17] A. Naeve, "A SECI-based framework for professional learning processes", Deliverable 10.1 of the ProLearn EU/FP6 Network of Excellence, IST 507310, August 2007.

[18] G. Paquette , "Graphical ontology modeling language for learning environments". Technology, Instruction, Cognition and Learning (TICL). vol. 5, no. 2/4, 2007.

[19] M-A. Sicilia, "Reuse, instructional design, and learning objects". Paper presented at Design-Based Approaches to Learning Objects and Learning Models Symposium Annual Conference of the American Educational Research Association (AERA), New York March 26, 2008.

[20] P. Charlton and G. Magoulas," "Self-configurable Framework for enabling Context-aware Learning Design". Proceedings of the IEEE International Conference on Intelligent Systems, pp. 1-6, 2010 . 